\title{
Articles
}

\section{Prediction of diabetes using ADA or WHO criteria in post-menopausal women: a 10-year follow-up study}

\author{
H. Larsson, F. Lindgärde, G. Berglund, B. Ahrén \\ Department of Medicine, Malmö University Hospital, Malmö, Sweden
}

\section{Abstract}

Aims/hypothesis. To study the risk of women with impaired fasting glucose (IFG) as against impaired glucose tolerance (IGT) developing diabetes.

Methods. Oral glucose tolerance tests $(75 \mathrm{~g})$ were done in 265 women selected at random at baseline (age 55-57 years) and at a 10-year follow-up. Of the women 42 had IFG/NGT (fasting glucose 6.1-6.9 mmol/1, 2-h glucose $<7.8 \mathrm{mmol} / \mathrm{l}), 66 \mathrm{IGT} /$ NFG (2-h glucose $7.8-11.0 \mathrm{mmol} / \mathrm{l}$, fasting glucose $<6.1 \mathrm{mmol} / \mathrm{l}), 30 \mathrm{IGT} / \mathrm{IFG}$ and $127 \mathrm{NFG/NGT}$. Results. The 10-year progression to diabetes was similar in IGT/NFG $(12.1 \%)$ and IFG/NGT groups $(11.9 \%, p=0.97)$. In IGT/IFG, $20.0 \%$ had developed diabetes, which was not significantly higher than in IFG/NGT and IGT/NFG $(p=0.53)$. In NFG/
NGT at baseline, only $3.9 \%$ had developed diabetes, which was lower than in the other groups $(p=0.023)$. Conclusion/interpretation. Fasting and 2-h glucose concentrations are equally good in predicting diabetes development over a 10-year period in Caucasian postmenopausal women. Because IGT is more common than IFG, measuring only fasting glucose concentrations would, however, result in missing a prediabetic stage in a large group of people at risk for diabetes and cardiovascular diseases. [Diabetologia (2000) 43: 1224-1228]

Keywords Impaired glucose tolerance, impaired fasting glucose, Type II diabetes, American Diabetes Association, World Health Organisation, progression, oral glucose tolerance test.

\section{Introduction}

Recent alterations in the criteria for diagnosis of diabetes by the American Diabetes Association (ADA) [1] have changed the focus of diabetes diagnostics from the oral glucose tolerance test to fasting glucose concentrations. A new category for impaired glucose regulation has also been introduced, impaired fasting glucose (IFG), characterized by slightly increased fasting glucose concentrations, above normal but be-

Received: 5 May 2000 and in revised form: 30 June 2000

Corresponding author: Dr. H. Larsson, Dept. of Medicine, Lund University, Malmö University Hospital, S-205 02 Malmö, Sweden

Abbreviations: ADA, American Diabetes Association; BG, blood glucose; NFG, normal fasting glucose; PG, plasma glucose; WHO, World Health Organisation. low the cut-off concentration for diabetes [1]. In contrast to the ADA criteria, the oral glucose tolerance test (OGTT) is recommended for diagnostic purposes by the World Health Organisation (WHO) both in their previous [2] and recent [3] recommendations. The OGTT identifies subjects with impaired glucose tolerance (IGT) having 2-h glucose concentrations above normal but below the cut-off concentration for diabetes. The relations between these two entities, IFG and IGT, have not been established, and there have been reports that subjects with IFG differ in clinical characteristics from subjects with IGT [4]. It has also been shown that the concordance between ADA and WHO criteria is low and that different subjects are diagnosed as diabetic by the two sets of criteria [5-10]. This is of importance also for the long-term risk for cardiovascular diseases because postprandial glycaemia seems to confer a higher risk than fasting hyperglycaemia [11-13]. Recently we re- 
Table 1. Anthropometric and metabolic characteristics of the study groups at the baseline examination between 1987 and 1991 and at the 10-year follow-up examination between 1998 and 1999

\begin{tabular}{lllll}
$\begin{array}{l}\text { IGT/NFG } \\
(n=66)\end{array}$ & P & $\begin{array}{l}\text { IFG/NGT } \\
(n=42)\end{array}$ & $\begin{array}{l}\text { IGT/IFG } \\
(n=30)\end{array}$ & $\begin{array}{l}\text { NFG/NGT } \\
(n=127)\end{array}$ \\
& IGT/NFG vs & & \\
\hline
\end{tabular}

Baseline variable

Body weight $(\mathrm{kg})$

Body mass index $\left(\mathrm{kg} / \mathrm{m}^{2}\right)$

Fasting blood glucose (mmol/l)

2-h blood glucose ( $\mathrm{mmol} / \mathrm{l})$

Follow-up variable

Body weight (kg)

Body mass index $\left(\mathrm{kg} / \mathrm{m}^{2}\right)$

Fasting plasma glucose $(\mathrm{mmol} / \mathrm{l})$

2-h plasma glucose ( $\mathrm{mmol} / \mathrm{l})$

$65.3 \pm 12.3$
$24.6 \pm 4.3$
$4.7 \pm 0.4$
$8.6 \pm 0.8$
$69.0 \pm 13.9$
$26.2 \pm 5.0$
$5.3 \pm 0.7$
$8.1 \pm 2.4$

$\begin{aligned} & 0.029 \\ & 0.089 \\ < & 0.001 \\ < & 0.001\end{aligned}$

0.056

0.14

0.027

0.16

$\begin{aligned} 71.1 & \pm 14.5 \\ 26.2 & \pm 4.8 \\ 5.5 & \pm 0.2 \\ 6.8 & \pm 0.9 \\ & \\ 74.5 & \pm 14.9 \\ 27.6 & \pm 5.1 \\ 5.7 & \pm 0.9 \\ 7.4 & \pm 2.7\end{aligned}$

$71.9 \pm 17.1$

$26.7 \pm 5.3$

$5.5 \pm 0.2$

$8.7 \pm 0.8$

$74.5 \pm 16.4$

$28.1 \pm 5.2$

$5.7 \pm 1.5$

$8.5 \pm 4.1$

\author{
$65.6 \pm 11.4$ \\ $24.4 \pm 3.9$ \\ $4.7 \pm 0.3$ \\ $6.4 \pm 0.9$ \\ $69.3 \pm 12.0$ \\ $26.1 \pm 4.4$ \\ $5.1 \pm 0.8$ \\ $6.3 \pm 2.0$
}

analysed a large group of glucose tolerance tests done between 1987 and 1991 [14]. We found that there was in fact very little overlap between the categories of IGT and IFG although the subjects who had IGT and those who had IFG had similar degrees of several risk factors for diabetes and cardiovascular diseases [15]. To study whether the risk of developing diabetes is similar in the two groups, we have now done a 10-year follow-up to find the progression to diabetes in subjects initially categorized as IGT or IFG.

\section{Subjects and methods}

Subjects. Between 1987 and 1991 oral glucose tolerance was measured in 1843 postmenopausal women aged 55-57 years, in a health screening programme as described previously [14]. To evaluate diabetes development over time in different glucose tolerance categories, a sample of 265 subjects selected at random from 1843 women was invited to a new oral glucose tolerance test in 1998-1999. The 1843 women were grouped according to WHO and ADA criteria [1-3] by their fasting and 2-h capillary blood glucose concentrations obtained from the baseline oral glucose tolerance test. The categories were: normal glucose tolerance according to both ADA and WHO criteria [normal fasting glucose(NFG)/NGT, fasting blood glucose $(\mathrm{BG})<5.3 \mathrm{mmol} / \mathrm{l}$ and 2 -h $\mathrm{BG}<7.8, n=1149]$, impaired glucose tolerance with normal fasting glucose (IGT/NFG, fasting $\mathrm{BG}<5.3 \mathrm{mmol} / \mathrm{l}$ and 2-h BG 7.8-11.0 mmol/l, $n=375)$, impaired fasting glucose with normal 2-h glucose (IFG/NGT, fasting BG 5.3-5.9 mmol/l and 2-h BG $<7.8 \mathrm{mmol} / \mathrm{l}, n=179$ ) and a combination of IGT/IFG (fasting BG 5.3-5.9 mmol/1 and 2-h BG 7.8-11.0 mmol/l, $n=140)$. The limit for fasting blood glucose was defined as $5.3 \mathrm{mmol} / 1$, which is equivalent to the criterion for plasma glucose concentrations suggested by ADA [1] using the $15 \%$ conversion factor as recommended by WHO [2]. A randomized sample of women from each of these groups was invited to take part in the follow-up study. Included in the follow-up study were 66 women with IGT/NFG, 42 women with IFG/NGT, 30 women with IGT/IFG and, as a control group, 127 women with NFG/NGT at the baseline examination. The women included in the follow-up study were representative of the groups they were selected from in body weight and glucose concentrations, except for the IGT/NFG group where the 66 women included had a slightly lower BMI than the original 375 women with IGT. The mean age at the follow-up study was $66 \pm 2.3$ years (mean \pm SD) and the mean follow-up time was 10 years 4 months \pm 1 year 10 months.

Follow-up oral glucose tolerance test. Oral glucose tolerance was measured after an overnight fast with a standard WHO 75-g glucose load [2], with venous plasma glucose (PG) samples taken before and $2 \mathrm{~h}$ after the glucose load. The subjects spent the $2 \mathrm{~h}$ in a semi-recumbent position. According to WHO and ADA criteria [1-3], follow-up glucose tolerance was categorized as normal, IGT and/or IFG, or diabetes according to the following cut-off values: normal was fasting PG less than $6.1 \mathrm{mmol} / \mathrm{l}$ and 2-h PG less than $7.8 \mathrm{mmol} / \mathrm{l}$; IFG and/or IGT was fasting PG $6.1-6.9 \mathrm{mmol} / \mathrm{l}$ and/or $2-\mathrm{h}$ PG 7.8-11.0 mmol/l; diabetes was fasting PG 7.0 or more and/or 2-h PG 11.1 or more $\mathrm{mmol} / \mathrm{l}$.

Statistical analyses. All statistical analyses were carried out with the SPSS for Windows system. Comparisons of diabetes development between the different groups were made with the Chi-squared test followed by the Bonferroni post hoc test for multiple comparisons.

\section{Results}

Body weight, BMI and fasting and 2-h glucose concentrations at the baseline and follow-up studies are shown in Table 1. Changes in glucose tolerance at the follow-up in the four categories are shown in Fig. 1. Diabetes development over the 10 years was $12.1 \%$ in the IGT/NFG group compared with $11.9 \%$ in the IFG/NGT group $(p=0.97)$. The group with IGT/IFG had a numerically higher diabetes development $(20.0 \%)$, which was not statistically significant (Chi-squared test $p=0.53$ ). Furthermore, in the IGT/NFG and IFG/NGT groups, approximately half of the subjects had normalized glucose tolerance over the 10 years, with no difference between the two groups (Chi-squared test $p=0.32$ ). In the group with NFG/NGT at baseline, glucose tolerance was maintained normal in the great majority of subjects and only $3.9 \%$ had developed diabetes. Thus, diabetes development over a 10-year period was higher in subjects with impaired glucose metabolism than in 

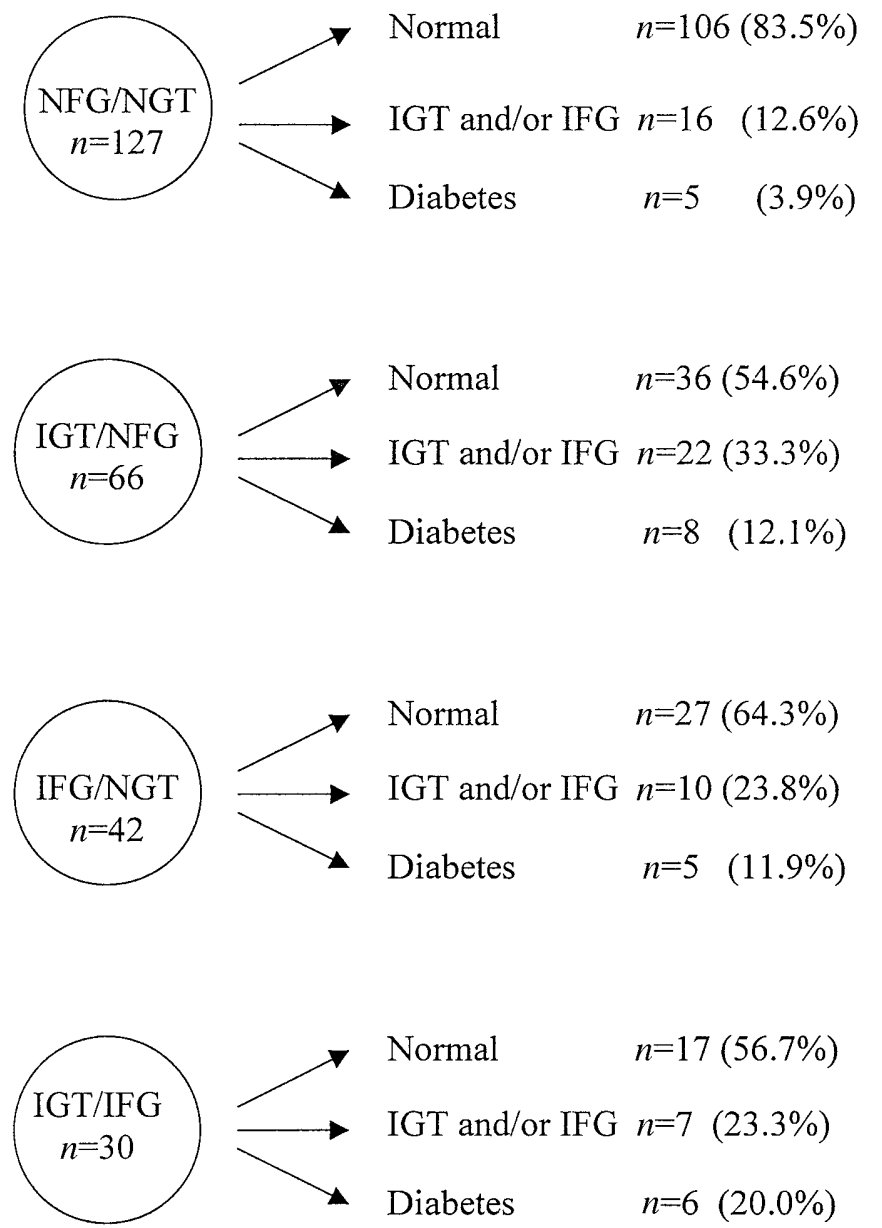

Fig. 1. Changes in glucose tolerance over 10 years in 265 women with normal glucose tolerance (NGT), impaired glucose tolerance (IGT/NFG), impaired fasting glucose (IFG/NGT) or a combination of impaired glucose tolerance and impaired fasting glucose (IGT/IFG) indicates number of subjects

the group with normal glucose metabolism (Chisquared test $p=0.023$ ). By using only the fasting glucose concentration for detection of people at risk for developing diabetes, only $46 \%$ of the cases would, however, have been detected compared with $79 \%$ when using an OGTT.

\section{Discussion}

We have previously presented data from Malmö, Sweden, that the prevalence of IGT is high in postmenopausal women in this cohort, yet the prevalence of diabetes is low [14]. We have also shown that in a subgroup of women in this large cohort, IGT and IFG represent two partially different groups [15]. This would suggest that OGTT is recommendable for diagnosis of impaired glucose metabolism, particularly if interventions are planned. The long-term development of diabetes in the two subgroups of IFG and IFG has not, however, been established. This 10-year follow-up study was therefore performed to determine whether progression to diabetes differs between subjects with IGT/NFG or IFG/NGT at the baseline examination. We found that the rate of diabetes development in both IGT/NFG and IFG/NGT groups over 10 years was low, slightly above $10 \%$, i.e. $1 \%$ per year and did not differ between IGT/ NFG and IFG/NGT subjects. Thus, in these postmenopausal women belonging to a group with a high prevalence of prediabetic conditions [14], the actual progression to diabetes was low, and did not depend on whether the subjects initially had increased fasting or 2-h glucose concentrations. This suggests that fasting and 2-h glucose concentrations are both of importance for detecting prediabetic people and that the two tests are equal for predicting diabetes. Restricting the detection to measurement of only the fasting glucose concentration will, however, miss the subjects in whom only post-load glucose concentrations are increased. Because the prevalence of IGT was higher in this group than the prevalence of IFG, choosing to measure only fasting glucose concentrations, i.e. excluding OGTT as recommended by ADA, would result in missing many subjects at risk of developing diabetes.

The progression rate to diabetes was lower in subjects with IGT or IFG in our study compared with previous reports from, for example, The Netherlands [16] and in Indians in South Africa [17] and among the Pima Indians [18], reporting a conversion rate to diabetes among subjects with IGT from 6 to $14 \%$ per year. It is probable that different ethnic groups have different characteristics in the progression rate to diabetes in IGT because other populations with low progression rates also exist, most notably in Scandinavia where published figures show conversion rates of approximately $2 \%$ per year [19-21].

The main finding of our study is that measuring only fasting glucose concentrations detects fewer prediabetic people than the oral glucose tolerance test. There have been similar findings in a study in Mauritius, where IFG was found to be less sensitive than IGT for predicting diabetes [22] and in a prospective Italian study [23]. Also several other studies have recently shown that measuring fasting glucose concentrations alone results in a lower prevalence of diabetes and failure to detect prediabetic subjects $[5-9,24$, 25]. This is a major drawback of the new recommendations, in view of the increasing awareness that postchallenge hyperglycaemia is not only a risk factor for diabetes development in itself but is also a major risk factor for cardiovascular diseases. For example, it was recently shown that an increased 2-h glucose concentration, in spite of a normal fasting glucose, is a risk factor for cardiovascular mortality, especially in women [11]. Other recent studies have suggested that IGT is in itself a cardiovascular risk factor whereas IFG is not [13]. Further, IFG was found not 
to be associated with atherosclerosis unless in combination with IGT [12]. Also total mortality was increased in subjects with IGT compared with subjects with IFG in a large European study [26]. In older subjects, the mortality risk was as high in subjects with isolated postchallenge hyperglycaemia as in diabetic subjects [27]. Therefore, the results of these studies suggest that it is vital also to detect the subjects with IGT alone.

It can be hypothesized that subjects with both increased fasting and 2-h glucose concentrations, IGT/ IFG, have a higher risk of developing diabetes. This group had a numerically higher progression rate $(20 \%)$ in our study but the difference was not statistically significant compared with subjects with either IGT or IFG alone. This could be due to lack of a true difference but could also be the result of lack of statistical power due to the smaller group of subjects with increases in both fasting and 2-h glucose concentrations at baseline in our study. A recent Italian study suggested, however, that the progression to diabetes was equally high in subjects with postload hyperglycaemia regardless of fasting glycaemia [23].

In this follow-up study of glucose tolerance in postmenopausal women we have shown that fasting and 2-h glucose concentrations are equally good in predicting diabetes development over a 10-year period and that IGT and IFG are two diagnostic entities which overlap only partially. In view of the greater number of subjects with IGT than IFG, we conclude that choosing to measure only fasting glucose concentrations would result in missing a prediabetic stage in a large group of people at risk for diabetes. It is important to detect this group because preventive measures are necessary to reduce the incidence of diabetes and its complications.

Acknowledgements. The authors are grateful to L. Bengtsson, L. Bryngelsson, U. Gustavsson, E. Holmström, K. Nilsson, M. Persson and I. Öhlund for expert technical assistance. The study was supported by the Swedish Medical Research Council (grant no 6834), Ernhold Lundström, Albert Påhlsson and Novo Nordic Foundations, the Swedish Diabetes Association, Malmö University Hospital and the Faculty of Medicine, Lund University.

\section{References}

1. American Diabetes Association (1998) Report of the expert committee on the diagnosis and classification of diabetes mellitus (Committee Report). Diabetes Care 21 [Suppl 1]: S5-S19

2. World Health Organization study Group on Diabetes Mellitus (1985) Technical Report series No 727. WHO, Geneva

3. Alberti KGMM, Zimmet PZ (1998) Definition, diagnosis and classification of diabetes mellitus and its complications. Part 1: Diagnosis and classification of diabetes mellitus. Provisional report of a WHO consultation. Diabet Med 15: 539-553
4. DECODE Study Group (1998) Will new diagnostic criteria for diabetes mellitus change phenotype of patients with diabetes? Reanalysis of European epidemiological data. BMJ 317: 371-375

5. De Vegt F, Dekker JM, Stehouwer CDA, Nijpels G, Bouter LM, Heine RJ (1998) The 1997 American Diabetes Association criteria versus the 1985 World Health Organization criteria for the diagnosis of abnormal glucose tolerance. Poor agreement in the Hoorn study. Diabetes Care 21: 1686-1690

6. Gimeno SGA, Ferreira SRG, Franco LJ, Iunes M (1998) Comparison of Glucose Tolerance Categories According to World Health Organization and American Diabetes Association Diagnostic Criteria in a Population-Based Study in Brazil. Diabetes Care 21: 1889-1892

7. Gomez-Pérez FJ, Aguilar-Salinas CA, López-Alvarenga JC, Perez-Jauregui J, Guillen-Pineda LE, Rull JA (1998) Lack of agreement between the World Health Organization category of impaired glucose tolerance and the American Diabetes Association category of impaired fasting glucose. Diabetes Care 21: 1886-1888

8. Richard JL, Vannereau D, Parer-Richard C (1998) Impact of the new ADA diagnostic criteria for diabetes on an obese population: Comparison with OGTT-based WHO criteria. Preliminary results. Diabetes Metab 24: 365-367

9. Wahl PW, Savage PJ, Psaty BM, Orchard TJ, Robbins JA, Tracy RP (1998) Diabetes in older adults: comparison of 1997 American Diabetes Association classification of diabetes mellitus with 1985 WHO classification. Lancet 352: 1012-1015

10. Lindahl B, Weinehall L, Asplund K, Hallmans G (1999) Screening for impaired glucose tolerance. Results from a population-based study in 21,057 individuals. Diabetes Care 22: 1988-1992

11. Barrett-Connor E, Ferrara A (1998) Isolated postchallenge hyperglycemia and the risk of fatal cardiovascular disease in older women and men. Diabetes Care 21: 1236-1239

12. Hanefeld M, Temelkova-Kurktschiev T, Schaper F, Henkel E, Siegert G, Koehler C (1999) Impaired fasting glucose is not a risk factor for atherosclerosis. Diabet Med 16: 212-218

13. Tominaga M, Eguchi H, Manaka H, Igarashi K, Kato T, Sekikawa A (1999) Impaired glucose tolerance is a risk factor for cardiovascular disease, but not impaired fasting glucose. The Funagata Diabetes Study. Diabetes Care 22: 920-924

14. Larsson H, Ahrén B, Lindgärde F, Berglund G (1995) Fasting blood glucose in determining the prevalence of diabetes in a large, homogeneous population of Caucasian middleaged women. J Intern Med 237: 537-541

15. Larsson H, Berglund G, Lindgärde F, Ahrén B (1998) Comparison of ADA and WHO criteria for diagnosis of diabetes and glucose intolerance (Letter). Diabetologia 41: 1124-1125

16. Nijpels G, Popp-Snijders C, Kostense PJ, Bouter LM, Heine RJ (1996) Fasting proinsulin and 2-h post-load glucose levels predict the conversion to NIDDM in subjects with impaired glucose tolerance: the Hoorn Study. Diabetologia 39: $113-118$

17. Motala AA, Omar MA, Gouws E (1993) High risk of progression to NIDDM in South-African Indians with impaired glucose tolerance. Diabetes 42: 556-563

18. Saad MF, Knowler WC, Pettitt DJ, Nelson RG, Mott DM, Bennett PH (1988) The natural history of impaired glucose tolerance in the Pimna Indians. N Engl J Med 319: 1500-1506 
19. Sartor G, Scherstén B, Carlström S, Melander A, Nordén $\AA$ A, Persson G (1980) Ten-year follow-up of subjects with impaired glucose tolerance: prevention of diabetes by tolbutamide and diet regulation. Diabetes 29: 41-49

20. Agner E, Thorsteinsson B, Eriksen M (1982) Impaired glucose tolerance and diabetes mellitus in elderly subjects. Diabetes Care 5: 600-604

21. Stengard JH, Pekkanen J, Tumoliehto J et al. (1993) Changes in glucose tolerance among elderly Finnisj men during a five-year follow-up: the Finnish cohorts of the Seven Countries Study. Diabetes Metab 19: 121-129

22. Shaw JE, Zimmet PZ, de Courten M et al. (1999) Impaired fasting glucose or impaired glucose tolerance. What best predicts future diabetes in Mauritius? Diabetes Care 22: 399-402

23. Vaccaro O, Ruffa G, Imperatore G, Iovino V, Rivellese AA, Riccardi G (1999) Risk of diabetes in the new diagnostic category of impaired fasting glucose: a prospective analysis. Diabetes Care 22: 1490-1493
24. Davies MJ, Muehlbayer S, Garrick P, McNally PG (1999) Potential impact of a change in the diagnostic criteria for diabetes mellitus on the prevalence of abnormal glucose tolerance in a local community at risk of diabetes: impact of new diagnostic criteria for diabetes mellitus. Diabet Med 16: 343-346

25. Mannucci E, Bardini G, Ognibene A, Rotella CM (1999) Comparison of ADA and WHO screening methods for diabetes mellitus in obese subjects. American Diabetes Association. Diabet Med 16: 579-585

26. DECODE Study Group. European Diabetes Epidemiology Group. Diabetes Epidemiology: Collaborative analysis Of Diagnostic criteria in Europe (1999) Glucose tolerance and mortality: comparison of WHO and American Diabetes Association diagnostic criteria. Lancet 354: 617-621

27. DECODE Study (Diabetes Epidemiology: Collaborative analysis of Diagnostic criteria in Europe) (1999) Consequences of the new diagnostic criteria for diabetes in older men and women. Diabetes Care 22: 1667-1671 